\title{
Equilibrium Distributions of Physical Clusters
}

\author{
Michael G. Mürmann \\ Institut für Angewandte Mathematik, Universität Heidelberg, \\ D-6900 Heidelberg, Federal Republic of Germany
}

\begin{abstract}
We consider classical systems of particles in $\mathbb{R}^{d}$ interacting by a stable pair potential with finite range. We are engaged in subdividing every particle configuration into clusters of interacting particles and studying the cluster distributions corresponding to equilibrium particle distributions.
\end{abstract}

\section{Introduction}

Let us consider an interaction in the $d$-dimensional Euclidean space $\mathbb{R}^{d}$ given by a pair potential $\Phi$, i.e., the potential energy of particles located at $x_{1}, \ldots, x_{n} \in \mathbb{R}^{d}$ is given by

$$
V\left(x_{1}, \ldots, x_{n}\right)=\sum_{1 \leqq i<j \leqq n} \Phi\left(x_{j}-x_{i}\right),
$$

where $\Phi: \mathbb{R}^{d} \rightarrow \mathbb{R} \cup\{+\infty\}$ is Lebesgue measurable with $\Phi(x)=\Phi(-x)$ for $x \in \mathbb{R}^{d}$. We suppose $\Phi$ to have the following properties:

stability: there exists $B \geqq 0$ with $V\left(x_{1}, \ldots, x_{n}\right) \geqq-n B$ for all $n$ and $x_{1}, \ldots, x_{n} \in \mathbb{R}^{d}$;

finite range: there exists $R>0$ with $\Phi(x)=0$ for $|x|>R$.

Because of the finite range property it is reasonable to introduce clusters of interacting particles. Thus a configuration $\left(x_{1}, \ldots, x_{l}\right)$ is a cluster, iff each two particles of the cluster interact at least indirectly.

This is a special type of physical clusters introduced in 1939 independently by Frenkel and Band in order to discuss condensation phenomena (see [3]). Recently Sinai [8] defined similar clusters - clusters in space-time however - for the existence of the time evolution of particle configurations.

Every finite or infinite particle configuration can now be subdivided into clusters with possibly infinite clusters defined in the same way. The purpose of this paper is to study the distribution of cluster configurations corresponding to equilibrium particle distributions in the sense of the DLR-equations in the case of only finite clusters with probability 1.

In Section 1 we give the exact definition of clusters by means of cluster functions and denote relations of these functions describing the subdivision of finite particle configurations into clusters. These relations are used in Section 2 to derive the cluster distribution corresponding to a grand canonical particle 
distribution of a bounded region. The result is a distribution, formally the same as a grand canonical distribution, if one introduces a certain measure on the cluster space and a hard core potential forbidding overlapping cluster configurations.

Section 3 treats the case of equilibrium particle distributions in $\mathbb{R}^{d}$. We give sufficient conditions for the absence of infinite clusters with probability 1 , the essential condition being a low activity condition. In this case we show that the validity of the DLR-equations of the particle distribution is equivalent to clusterDLR-equations of the corresponding cluster distributions with the abovementioned measure and hard core potential.

In Section 4 we reverse the point of view and start from cluster configurations. We prove an existence theorem of cluster distributions satisfying the cluster-DLRequations under the low activity condition. As a corollary we get an existence theorem of the corresponding particle distributions. Remark that concerning the behaviour of the potential for small distances it only presumes stability. We close in Section 5 with some remarks concerning the connection between the uniqueness of the equilibrium distribution and the absence of infinite clusters.

Acknowledgement. I want to thank Professor J. Groeneveld for helpful discussions on the formal resemblance of these clusters with the Mayer clusters and how to use it.

\section{Physical Clusters}

The notion of clusters initiated in the introduction is equivalent to saying that a particle configuration is a cluster iff the graph obtained by joining interacting particles is connected. We use this fact to derive an explicit expression for a cluster function $u$ defined on all finite non-empty configurations, which is 1 for clusters and 0 otherwise.

For $x, y \in \mathbb{R}^{d}$ we set

$$
h(x, y)=\left\{\begin{array}{lll}
1 & \text { for } & |x-y|>R \\
0 & \text { for } & |x-y| \leqq R
\end{array}\right.
$$

and for $x_{1}, \ldots, x_{n}, y_{1}, \ldots, y_{m} \in \mathbb{R}^{d}$

$$
h\left(x_{1}, \ldots, x_{n} ; y_{1}, \ldots, y_{m}\right)=\prod_{i=1}^{n} \prod_{j=1}^{m} h\left(x_{i}, y_{j}\right) .
$$

This function is the usual function $h$ as defined in the theory of the Mayer expansion ([2]) related to the pair potential $\varphi$ given by:

$$
\varphi(x)=\left\{\begin{array}{lll}
0 & \text { for } & |x|>R \\
+\infty & \text { for } & |x| \leqq R
\end{array}\right.
$$

The cluster function $u$ is now defined on the topological sum $\sum_{l=1}^{\infty}\left(\mathbb{R}^{d}\right)^{l}$ representing the space of all finite non-empty configurations in the following way:

$$
u\left(x_{1}, \ldots, x_{l}\right)=\sum_{G \in C_{l}} \prod_{(i, j) \in G}\left(1-h\left(x_{i}, x_{j}\right)\right) \prod_{(a, b) \notin G} h\left(x_{a}, x_{b}\right),
$$

where $C_{l}$ is the set of all connected graphs $G$ with vertices $1, \ldots, l$. 
Obviously this is the desired function. It resembles the usual Ursell function $U$ related to $\varphi$

$$
U\left(x_{1}, \ldots, x_{l}\right)=\sum_{G \in C_{l}} \prod_{(i, j) \in G}\left(h\left(x_{i}, x_{j}\right)-1\right) .
$$

We shall make use of this resemblance by introducing $t$-functions which satisfy $u-t$ - and $t-u$-relations. Comparing them with the corresponding $U-T$ - and $T-U$-relations ([2], Section IV.C) we finally get an estimation of $u$ by $U$, which we shall need later.

The $t$-functions are defined in the same way as the $T$-functions by replacing $U$ by $u$. For the special type of $t$-functions we only need one can take the $t-u$ relations directly as definition:

$$
t\left(x ; x_{1}, \ldots, x_{l}\right)=-f\left(x ; x_{1}, \ldots, x_{l}\right) u\left(x_{1}, \ldots, x_{l}\right)
$$

with $f\left(x ; x_{1}, \ldots, x_{l}\right)=h\left(x ; x_{1}, \ldots, x_{l}\right)-1$.

For the $u$-t-relations we use the customary abbreviation $u(\gamma)=u\left(x_{i_{1}}, \ldots, x_{i_{1}}\right)$ for every finite set $\gamma=\left\{i_{1}, \ldots, i_{l}\right\}$ of non-negative integers. Because of the symmetry of $u$ this is uniquely defined. The analogous abbreviation will be used for other symmetric functions, too. With this notation the $u-t$-relations are:

$$
u(\{1,2, \ldots, l\})=\sum_{\delta c \gamma} t(\{1\} ;\{2\} \cup \delta) u(\{1\} \cup \gamma \backslash \delta) h(\{2\} \cup \delta ; \gamma \backslash \delta)
$$

with $\gamma=\{3, \ldots, l\}$.

The proof of (3) is almost the same as for the $U-T$-relations. Thus it is sufficient to show how the difference between the definition of $u$ and $U$ leads to different relations.

The $T-U$-relations are ([2], IV (39))

$$
T\left(x ; x_{1}, \ldots, x_{l}\right)=f\left(x ; x_{1}, \ldots, x_{l}\right) \quad U\left(x_{1}, \ldots, x_{l}\right)
$$

and the $U-T$-relations ([2], IV (37))

$$
U(\{1,2, \ldots, l\})=\sum_{\delta \subset \gamma} T(\{1\} ;\{2\} \cup \delta) U(\{1\} \cup \gamma \backslash \delta)
$$

with $\gamma=\{3, \ldots, l\}$.

The replacement of $f$ by $-f$ in the definition of $u$ leads to the same replacement in (2), and the additional $h$-term leads to the additional $h$-term in (3).

Comparing the $t-u$ - and the $u-t$-relations with the $T-U$ - and $U-T$-relations there follows by induction on $l$, since $u(\{1\})=U(\{1\})=1$ holds, the following lemma:

Lemma 1.1. For $l \geqq 1$ and $x, x_{1}, \ldots, x_{l} \in \mathbb{R}^{d}$ :

$$
\begin{aligned}
& 0 \leqq u\left(x_{1}, \ldots, x_{l}\right) \leqq(-1)^{l-1} U\left(x_{1}, \ldots, x_{l}\right) \\
& 0 \leqq t\left(x ; x_{1}, \ldots, x_{l}\right) \leqq(-1)^{l} T\left(x ; x_{1}, \ldots, x_{l}\right) .
\end{aligned}
$$

Let us introduce now the space of clusters. The space $\mathscr{C}_{l}$ of $l$-clusters is given by

$$
\mathscr{C}_{l}=\left\{\left(x_{1}, \ldots, x_{l}\right): u\left(x_{1}, \ldots, x_{l}\right)=1\right\} \subset\left(\mathbb{R}^{d}\right)^{l}
$$

and the space $\mathscr{C}$ of all clusters by

$$
\mathscr{C}=\sum_{l=1}^{\infty} \mathscr{C}_{l}=u^{-1}(\{1\}) .
$$

In the following we shall designate clusters by capital letters as $X, Y, Z$. 
For the subdivision of finite configurations $\left(x_{1}, \ldots, x_{n}\right)$ into clusters one easily sees that

$$
\sum_{\gamma \subset\{2, \ldots, n\}} u(\{1\} \cup \gamma) h(\{1\} \cup \gamma ;\{2, \ldots, n\} \backslash \gamma) \equiv 1 \quad \text { on } \quad\left(\mathbb{R}^{d}\right)^{n}
$$

which implies

$$
\sum^{(n)} k !^{-1} u\left(\gamma_{1}\right) \ldots u\left(\gamma_{k}\right) \prod_{1 \leqq i<j \leqq k} h\left(\gamma_{i} ; \gamma_{j}\right) \equiv 1 \quad \text { on } \quad\left(\mathbb{R}^{d}\right)^{n},
$$

where the sum $\sum^{(n)}$ extends over all ordered partitions $\left(\gamma_{1}, \ldots, \gamma_{k}\right)$ of $\{1, \ldots, n\}$ into an arbitrary number of disjoint non-empty subsets. Relation (4) relates to the subdivision of $n$-particle configurations into clusters. The empty particle configuration $\left(\mathbb{R}^{d}\right)^{0}$ is attached an empty cluster configuration $\mathscr{C}^{0}$.

The $h$-term in (4) excludes cluster configurations which overlap in the sense that at least two different clusters can be jointed to a new cluster. We define a corresponding function $H$ on the space $\sum_{k=0}^{\infty} \mathscr{C}^{k}$ of all finite cluster configurations by

$$
\begin{aligned}
& \left.H\right|_{\mathscr{C}^{0}} \equiv 1 \\
& \left.H\right|_{\mathscr{C}^{1}} \equiv 1 \\
& H\left(\left(x_{1}, \ldots, x_{l}\right),\left(y_{1}, \ldots, y_{m}\right)\right)=h\left(x_{1}, \ldots, x_{l} ; y_{1}, \ldots, y_{m}\right) \\
& H\left(X_{1}, \ldots, X_{k}\right)=\sum_{1 \leqq i<j \leqq k} H\left(X_{i}, X_{j}\right) \text { for } k \geqq 2 .
\end{aligned}
$$

It can be conceived as related to a hard core potential of cluster configurations excluding overlapping clusters.

\section{Grand Canonical Cluster Distributions}

In order to introduce the method how cluster distributions are derived from particle distributions we treat the case of the grand canonical distribution in some detail.

Let $z>0$ be the activity and $\beta>0$ be the inverse temperature parameter. Then for a bounded Borel set $\Lambda \subset \mathbb{R}^{d}$ the grand canonical partition function is given by

$$
\mathscr{Z}(\Lambda)=\sum_{n=0}^{\infty} n !^{-1} z^{n} \int \ldots \int_{\Lambda^{n}} e^{-\beta V\left(x_{1}, \ldots, x_{n}\right)} d x_{1} \ldots d x_{n} .
$$

Since there is no interaction between non-overlapping clusters relation (4) yields:

$$
e^{-\beta V\left(x_{1}, \ldots, x_{n}\right)}=\sum^{(n)} k !^{-1} u\left(\gamma_{1}\right) e^{-\beta V\left(\gamma_{1}\right)} \ldots u\left(\gamma_{k}\right) e^{-\beta V\left(\gamma_{k}\right)} \prod_{1 \leqq i<j \leqq k} h\left(\gamma_{i} ; \gamma_{j}\right)
$$

with $V(\gamma)$ defined in the known way.

Thus:

$$
\begin{aligned}
\mathscr{Z}(\Lambda)= & 1+\sum_{n=1}^{\infty} n !^{-1} z^{n} \int \ldots \int_{\Lambda^{n}} \sum^{(n)} k !^{-1} u\left(\gamma_{1}\right) e^{-\beta V\left(\gamma_{1}\right)} \ldots u\left(\gamma_{k}\right) e^{-\beta V\left(\gamma_{k}\right)} \\
& \cdot \prod_{1 \leqq i<j \leqq k} h\left(\gamma_{i} ; \gamma_{j}\right) d x_{1} \ldots d x_{n} \\
= & 1+\sum_{n=1}^{\infty} z^{n} \int \ldots \int_{\Lambda^{n}} \sum^{\prime(n)}\left(k !\left|\gamma_{1}\right| ! \ldots\left|\gamma_{k}\right| !\right)^{-1} u\left(\gamma_{1}\right) e^{-\beta V\left(\gamma_{1}\right)} \ldots u\left(\gamma_{k}\right) e^{-\beta V\left(\gamma_{k}\right)} \\
& \cdot \prod_{1 \leqq i<j \leqq k} h\left(\gamma_{i} ; \gamma_{j}\right) d x_{1} \ldots d x_{n} .
\end{aligned}
$$


The prime on the sum indicates that it only extends over the ordered partitions of $\{1, \ldots, n\}$ into successive non-empty sections. If we now set

$$
\Lambda_{*}=\left\{X=\left(x_{1}, \ldots, x_{l}\right): x_{i} \in \Lambda \text { for } i=1, \ldots, l ; l \geqq 1\right\}
$$

and define the measure $\mu$ on $\mathscr{C}$ by

$$
d \mu_{\mathscr{C}_{l}}\left(x_{1}, \ldots, x_{l}\right)=l !^{-1} z^{l} e^{-\beta V\left(x_{1}, \ldots, x_{l}\right)} d x_{1} \ldots d x_{l}
$$

then we finally get

$$
\mathscr{Z}(\Lambda)=\sum_{k=0}^{\infty} k !^{-1} \int \ldots \int_{\Lambda_{k}^{k}} H\left(X_{1}, \ldots, X_{k}\right) d \mu\left(X_{1}\right) \ldots d \mu\left(X_{k}\right)=3\left(\Lambda_{*}\right)
$$

with $3(A)$ defined for every Borel set $A \subset \mathscr{C}$ by

$$
3(A)=\sum_{k=0}^{\infty} k !^{-1} \int \ldots \int_{A^{k}} H\left(X_{1}, \ldots, X_{k}\right) d \mu\left(X_{1}\right) \ldots d \mu\left(X_{k}\right) \leqq \infty .
$$

The distribution of the cluster configurations corresponding to the grand canonical distribution can now easily be derived in the same way. The only problem is that to a fixed particle configuration there belongs no unique labelling of the clusters and thus no unique probability measure on $\sum_{k=0}^{\infty} \Lambda_{*}^{k}$ as cluster distribution. We naturally take the symmetric version ${ }^{1}$ with restriction on $\Lambda_{*}^{k}$ given by:

$$
3\left(\Lambda_{*}\right)^{-1} k !^{-1} H\left(X_{1}, \ldots, X_{k}\right) d \mu\left(X_{1}\right) \ldots d \mu\left(X_{k}\right) .
$$

This distribution is formally equal to a grand canonical distribution by replacing the Lebesgue measure by the measure $\mu$ and introducing the potential of cluster configurations mentioned at the end of the last section. It also has an interesting meaning from the point of view of stochastic point processes. If $H$ is replaced by the constant 1 , we would get the Poisson point process with respect to the measure $\mu$ restricted to $\Lambda_{*}$. The function $H$ however excludes certain configurations. So we can conceive the distribution as "the Poisson point process with respect to the measure $\mu_{A}$, and exclusion function $H$ ". It is in fact the conditional distribution of the Poisson point process under the condition of non-overlapping configurations.

We finish this section with some properties of 3 .

Lemma 2.1. Let $A, B \subset \mathscr{C}$ be Borel sets. Then:

(i) $1+\mu(A) \leqq 3(A) \leqq e^{\mu(A)}$.

(ii) $A \subset B \Rightarrow 3(A) \leqq 3(B)$.

(iii) $A \cap B=\emptyset \Rightarrow 3(A \cup B) \leqq 3(A) 3(B)$.

(iv) $A \cap B=\emptyset, \eta=\mu(A \cap\{X: H(X, Z)=0$ for at least one $Z \in B\})$ $\Rightarrow e^{-\eta} \mathfrak{Z}(A) \mathcal{Z}(B) \leqq 3(A \cup B)$.

Proof. (i) and (ii) are trivial.

(iii) and (iv): For $A \cap B=\emptyset$ we have:

$$
\begin{aligned}
3(A \cup B)= & \sum_{k=0}^{\infty} k !^{-1} \int \ldots \int_{(A \cup B)^{k}} H\left(X_{1}, \ldots, X_{k}\right) d \mu\left(X_{1}\right) \ldots d \mu\left(X_{k}\right) \\
= & \sum_{i=0}^{\infty} i !^{-1} \sum_{j=0}^{\infty} j !^{-1} \int \ldots \int_{A^{i}} \int \ldots \int_{B^{j}} H\left(Y_{1}, \ldots, Y_{i}, Z_{1}, \ldots, Z_{j}\right) \\
& \cdot d \mu\left(Y_{1}\right) \ldots d \mu\left(Y_{i}\right) d \mu\left(Z_{1}\right) \ldots d \mu\left(Z_{j}\right) .
\end{aligned}
$$

Another possibility is to go over to the symmetrized spaces, which we shall do in the next section. 
The inequality $H\left(Y_{1}, \ldots, Y_{i}, Z_{1}, \ldots, Z_{j}\right) \leqq H\left(Y_{1}, \ldots, Y_{i}\right) H\left(Z_{1}, \ldots, Z_{j}\right)$ yields (iii).

In the case $A \cap\{X: H(X, Z)=0$ for at least one $Z \in B\}=\emptyset$ we have

$$
H\left(Y_{1}, \ldots, Y_{i}, Z_{1}, \ldots, Z_{j}\right)=H\left(Y_{1}, \ldots, Y_{i}\right) H\left(Z_{1}, \ldots, Z_{j}\right)
$$

from which there follows $3(A \cup B)=3(A) 3(B)$.

In the general case we set $A_{1}=A \cap\{X: H(X, Z)=0$ for at least one $Z \in B\}$ and $A_{2}=A \backslash A_{1}$. Then we have:

$$
3\left(A_{2}\right) 3(B)=3\left(A_{2} \cup B\right) \leqq 3(A \cup B)
$$

and

$$
3(A) \leqq 3\left(A_{1}\right) 3\left(A_{2}\right) \leqq e^{\mu\left(A_{1}\right)} 3\left(A_{2}\right)
$$

which yields (iv).

\section{Equilibrium Cluster Distributions}

We briefly state the notions concerning infinite particle configurations and equilibrium distributions satisfying the DLR-equations.

Let $\mathscr{X}$ be the set of all locally finite labelled configurations in $\mathbb{R}^{d}$, i.e. the set of all finite and infinite sequences $\left(x_{i}\right)$ with elements $x_{i} \in \mathbb{R}^{d}$ such that every compact subset of $\mathbb{R}^{d}$ contains only finitely many members of the sequence. Setting two such sequences to be equivalent, if they only differ by a permutation of their indices, we denote by $[\mathscr{X}]$ the set of all equivalence classes as the set of all locally finite non-labelled configurations in $\mathbb{R}^{d}$. The equivalence class belonging to $\left(x_{i}\right) \in \mathscr{X}$ will be denoted by $\left[\left(x_{i}\right)\right]$. The set $[\mathscr{X}]$ is equipped with the weak topology with respect to the set of all functions $\sum_{i} f\left(x_{i}\right), f$ running over all continuous functions with compact support. In this topology $[\mathscr{X}]$ is a Polish space ([5]).

For each Borel set $B \subset \mathbb{R}^{d}$ let $\mathscr{X}_{B}$ be the subset of sequences in $\mathscr{X}$ with elements in $B$ and $\left[\mathscr{X}_{B}\right]$ be the set of the corresponding equivalence classes. Then the mapping $\pi_{B}:[\mathscr{X}] \rightarrow\left[\mathscr{X}_{B}\right]$, which cancels all elements of a configuration outside $B$, is measurable with respect to the $\sigma$-algebra $\mathfrak{B}$ resp. $\mathfrak{B}_{B}$ of the Borel sets of $[\mathscr{X}]$ resp. $\left[\mathscr{X}_{B}\right]$. The mapping

$$
\pi_{B} \times \pi_{C B}:[\mathscr{X}] \rightarrow\left[\mathscr{X}_{B}\right] \times\left[\mathscr{X}_{C B}\right]
$$

yields an identification of the measurable spaces $([\mathscr{X}], \mathfrak{B})$ and $\left(\left[\mathscr{X}_{B}\right], \mathfrak{B}_{B}\right) \times$ $\left(\left[\mathscr{X}_{C B}\right], \mathfrak{B}_{C B}\right)$. If $B \subset \mathbb{R}^{d}$ is a bounded Borel set then $\mathscr{X}_{B}=\sum_{n=0}^{\infty} B^{n}$ and $\left[\mathscr{X}_{B}\right]=$ $\sum_{n=0}^{\infty}\left(B^{n}\right)_{\text {symm }}$.

Furthermore we use the following denotation: Let $B \subset \mathbb{R}^{d}$ be a bounded Borel set and $\lambda_{B}$ be the Lebesgue measure on $B$. On $\mathscr{X}_{B}=\sum_{n=0}^{\infty} B^{n}$ we have the measure whose restriction on $B^{n}$ is $n !^{-1} \lambda^{\otimes n}$. Then we call the corresponding measure on $\left[\mathscr{X}_{B}\right]$ the non-normalized Poisson measure $h\left(\lambda_{B}\right)$.

For every bounded Borel set $\Lambda \subset \mathbb{R}^{d}$ and every configuration $\left[\left(y_{i}\right)\right] \in\left[\mathscr{X}_{\mathrm{C}_{A}}\right]$ the conditional Gibbs distribution of particles in $\Lambda$ under the outer configuration $\left[\left(y_{i}\right)\right]$ is the probability measure $P_{\Lambda \mid\left[\left(y_{1}\right)\right]}$ on $\left[\mathscr{X}_{A}\right]$ with the density

$$
\begin{aligned}
& p_{\Lambda \mid\left[\left(y_{i}\right)\right]}\left(\left[\left(x_{1}, \ldots, x_{n}\right)\right]\right) \\
& \quad=\mathscr{Z}\left(\Lambda \mid\left[\left(y_{i}\right)\right]\right)^{-1} \cdot z^{n} \exp \left\{-\beta V\left(x_{1}, \ldots, x_{n}\right)-\beta \sum_{i} \sum_{k=1}^{n} \Phi\left(y_{i}-x_{k}\right)\right\}
\end{aligned}
$$


with respect to the measure $\left.h_{(} \lambda_{A}\right)$. Evidently this expression for the density does not depend on the representatives of $\left[\left(x_{1}, \ldots, x_{n}\right)\right]$ and $\left[\left(y_{i}\right)\right]$ and thus is uniquely defined.

$\mathscr{Z}\left(\Lambda \mid\left[\left(y_{i}\right)\right]\right)$ is the obvious normalization factor, called the conditional partition function.

An equilibrium particle distribution is now defined as a probability measure $P$ on $[\mathscr{X}]$ which satisfies the DLR-equations ([7]):

For every bounded Borel-set $\Lambda \subset \mathbb{R}^{d}$ and every $f \in L^{1}([\mathscr{X}], P)$

$$
\int_{[\mathscr{C}]} f d P=\int_{\left[\mathscr{X}_{[\Lambda]}\right]} d \pi_{C_{\Lambda}}(P)\left(\left[\left(y_{i}\right)\right]\right) \int_{\left[\mathscr{X}_{\Lambda}\right]} d P_{\Lambda \mid\left[\left(y_{i}\right)\right]} f\left(\left[\left(x_{1}, \ldots, x_{n}\right)\right] \times\left[\left(y_{i}\right)\right]\right)
$$

holds with the above mentioned identification.

The DLR-equations signify that $P_{\Lambda \mid\left[\left(y_{2}\right)\right]}$ is one version of the conditional distribution of particles in $\Lambda$ under the outer configuration $\left[\left(y_{i}\right)\right]$.

We now pass over to cluster distributions corresponding to equilibrium particle distributions.

The subdivision of infinite particle configurations into clusters can only be performed in the space of non-labelled configurations. The formula resembles (4) with the sum extending over all non-ordered partitions and without the factor $k !^{-1}$. We shall not use it explicitely, however.

Let us first treat the problem of the absence of infinite clusters with probability 1 . It will turn out that the essential condition is the following. If we set for every Borel set $\Lambda \subset \mathbb{R}^{d}$ :

$$
\Lambda^{*}=\left\{X=\left(x_{1}, \ldots, x_{l}\right): x_{i} \in \Lambda \text { for at least one } i ; l \geqq 1\right\}
$$

then the condition is

$$
\mu\left(\Lambda^{*}\right)<\infty \text { for every bounded Borel set } \Lambda \subset \mathbb{R}^{d} \text {. }
$$

Because of Lemma 2.1 i, (13) is equivalent to

$$
3\left(\Lambda^{*}\right)<\infty \text { for every bounded Borel set } \Lambda \subset \mathbb{R}^{d} \text {. }
$$

We shall show below, that the radius of convergence of $\mu\left(\Lambda^{*}\right)$ being a power series in $z$ does not depend on $\Lambda$ and is strictly positive. Thus (13) holds for sufficiently small $z$. Before that we prove the following theorem.

Theorem 3.1. Let (13) be satisfied

i) If $P$ is a limit of grand canonical distributions, then there exist only finite clusters $P$-a.s.

ii) For a non-negative potential $\Phi \geqq 0$ there exist only finite clusters $P$-a.s. for every equilibrium distribution $P$.

The proof of this theorem uses the following lemma, which we shall need later, too.

Lemma 3.2. Let $(E, \mathscr{E})$ be an arbitrary measurable space, and let $v$ be a symmetric measure on $\left(\sum_{n=0}^{\infty} E^{n}, \sigma\left(\sum_{n=0}^{\infty} \mathscr{E}^{n}\right)\right)$ with restriction $n !^{-1} v_{n}$ on $\left(E^{n}, \mathscr{E}^{n}\right)$. Let $A \in \mathscr{E}$ and

$$
A^{*}=\left\{\left(x_{1}, \ldots, x_{n}\right): x_{i} \in A \text { for at least one } i ; n \geqq 1\right\} \text {. }
$$

Then

$$
\sum_{n=0}^{\infty}(n+1) !^{-1} v_{n+1}\left(A \times E^{n}\right) \leqq v\left(A^{*}\right) \leqq \sum_{n=0}^{\infty} n !^{-1} v_{n+1}\left(A \times E^{n}\right) .
$$


Proof. Call

$$
\begin{aligned}
& \begin{array}{l}
A_{n}^{*}=\left\{\left(x_{1}, \ldots, x_{n}\right): x_{i} \in A \text { for at least one } i\right\} \\
=\bigcup_{i=1}^{n}\left\{\left(x_{1}, \ldots, x_{n}\right): x_{i} \in A\right\}
\end{array} \\
& \begin{aligned}
& v\left(A^{*}\right)=\sum_{n=1}^{\infty} n !^{-1} v_{n}\left(A_{n}^{*}\right) \\
& v_{n}\left(\left\{\left(x_{1}, \ldots, x_{n}\right): x_{1} \in A\right\}\right) \leqq v_{n}\left(\bigcup_{i=1}^{n}\left\{\left(x_{1}, \ldots, x_{n}\right): x_{i} \in A\right\}\right) \\
& \leqq \sum_{i=1}^{n} v_{n}\left(\left\{\left(x_{1}, \ldots, x_{n}\right): x_{i} \in A\right\}\right)=n \cdot v_{n}\left(A \times E^{n-1}\right)
\end{aligned}
\end{aligned}
$$

which yields the result.

Proof of Theorem 3.1. We first prove Part ii).

Let $\Lambda \subset \mathbb{R}^{d}$ be a bounded Borel set and $\left[\left(y_{i}\right)\right] \in\left[\mathscr{X}_{\mathrm{C}_{A}}\right]$. We regard the conditional Gibbs distribution $P_{\Lambda \mid\left[\left(y_{i}\right)\right]}$, subdivide every particle configuration in $\Lambda$ into clusters regardless eventual clustering with outer particles. The result is the probability measure $\bar{Q}_{\Lambda_{*} \mid\left[\left(y_{i}\right)\right]}$ on $\sum_{n=0}^{\infty} \Lambda_{*}^{n}$ with density

$$
\bar{q}_{\Lambda_{*} \mid\left[\left(y_{i}\right)\right]}\left(\left[\left(X_{1}, \ldots, X_{k}\right)\right]\right)=\overline{3}\left(\Lambda_{*} \mid\left[\left(y_{i}\right)\right]\right)^{-1} k !^{-1} H\left(X_{1}, \ldots, X_{k}\right) \prod_{m=1}^{k} \prod_{i} e^{-W\left(X_{m}, y_{i}\right)}
$$

with respect to the non-normalized Poisson-measure $\not\left(\mu_{\Lambda_{*}}\right)$ defined in the same way as $f\left(\lambda_{B}\right)$. We used the notation

$$
W(X, y)=\sum_{i=1}^{l} \Phi\left(x_{i}-y\right) \text { for } X=\left(x_{1}, \ldots, x_{l}\right)
$$

and the normalization factor $\overline{3}\left(\Lambda_{*} \mid\left[\left(y_{i}\right)\right]\right)$.

Let $\varepsilon>0$ and $N$ be such that $\mu\left(\Lambda_{\geqq N}^{*}\right) \leqq \varepsilon$ with $\Lambda_{\geqq N}^{*}=\bigcup_{n=N}^{\infty} \Lambda^{*} \cap \mathscr{C}_{n}$. This is possible because of (13). Furthermore let $\Lambda \subset \Lambda^{\prime} \subset \mathbb{R}^{d}$ be a bounded Borel set and let $\left[\left(y_{i}\right)\right] \in\left[\mathscr{X}_{\left[\Lambda^{\prime}\right.}\right]$.

Then using Lemma 3.2 there follows:

$$
\begin{aligned}
& \bar{Q}_{\Lambda^{\prime} \mid\left[\left(y_{i}\right)\right]}\left(\left\{\left[\left(X_{1}, \ldots, X_{k}\right)\right]: X_{m} \in \Lambda_{\geqq N}^{*} \text { for at least one } m, k \geqq 1\right\}\right) \\
& \leqq \overline{3}\left(\Lambda_{*}^{\prime} \mid\left[\left(y_{i}\right)\right]\right)^{-1} \sum_{k=0}^{\infty} k !^{-1} \int_{\Lambda_{\geqq N}^{*} \cap \Lambda^{\prime}} \int \ldots \int_{\Lambda^{\prime} k} H\left(X_{0}, X_{1}, \ldots, X_{k}\right) \\
& \cdot \prod_{m=0}^{k} \prod_{i} e^{-\beta W\left(X_{m}, y_{2}\right)} d \mu\left(X_{0}\right) d \mu\left(X_{1}\right) \ldots d \mu\left(X_{k}\right) \\
& \leqq \overline{3}\left(\Lambda_{*}^{\prime} \mid\left[\left(y_{i}\right)\right]\right)^{-1} \sum_{k=0}^{\infty} k !^{-1} \int_{\Lambda_{\geqq N}^{*} \cap \Lambda_{*}^{\prime}}^{\infty} \int \ldots \int_{\Lambda^{\prime} k} H\left(X_{1}, \ldots, X_{k}\right) \\
& \cdot \prod_{m=1}^{k} \prod_{i} e^{-\beta W\left(X_{m}, y_{i}\right)} d \mu\left(X_{0}\right) d \mu\left(X_{1}\right) \ldots d \mu\left(X_{k}\right) \\
&= \mu\left(\Lambda_{\geqq N}^{*} \cap \Lambda_{*}^{\prime}\right) \leqq \varepsilon .
\end{aligned}
$$

This holds for all such $\Lambda^{\prime}$ and $\left[\left(y_{i}\right)\right] \in\left[\mathscr{X}_{C \Lambda^{\prime}}\right]$. Fix $\Lambda^{\prime}$ now with $\operatorname{dist}\left(\Lambda, C \Lambda^{\prime}\right)>$ $(N-1) R$. Then no particle outside $\Lambda^{\prime}$ can interact with a particle belonging to a cluster of $\Lambda_{<N}^{*}:=\Lambda^{*} \cap \bigcup_{n=1}^{N-1} \mathscr{C}_{n}$.

Thus the DLR-equations and the just proved inequality yield:

$P(\{$ all particles of $\Lambda$ cluster only with finitely many other particles $\})$

$\geqq P(\{$ all particles of $\Lambda$ cluster with at most $(N-1)$ particles $\}) \geqq 1-\varepsilon$.

Since this holds for every $\varepsilon>0$ there follows:

$P(\{$ all particles of $\Lambda$ cluster only with finitely many other particles $\})=1$.

Taking an increasing sequence $\Lambda_{n} \uparrow \mathbb{R}^{d}$ the result follows. 
Part i) is proved in the same way. Let $P$ be a limit of grand canonical distributions $P_{m}$ belonging to an increasing sequence $\Lambda_{m}^{\prime} \uparrow \mathbb{R}^{d}$.

If we take for a bounded Borel set $\Lambda$ and $m$ such that $\Lambda \subset \Lambda_{m}^{\prime}$ in the proof of ii) $P_{m}$ instead of $P_{A_{m}^{\prime} \mid\left[\left(y_{i}\right)\right]}$ then we get the result similarly.

Proposition 3.3. The radius of convergence of $\mu\left(\Lambda^{*}\right)$ as a power series in $z$ does not depend on $\Lambda$ and is the same as of the following series not depending on $x$ :

$$
\sum_{l=0}^{\infty} l !^{-1} z^{l} \int \ldots \int_{\left(\mathbb{R}^{d}\right)^{l}} e^{-\beta V\left(x, x_{1}, \ldots, x_{l}\right)} u\left(x, x_{1}, \ldots, x_{l}\right) d x_{1} \ldots d x_{l} .
$$

It is $\geqq\left(e^{\beta B+1} R^{d} V_{d}\right)^{-1}$ with $V_{d}$ being the volume of the d-dimensional unit ball and for non-negative potentials even $\geqq\left(e \int_{|x| \geqq R} e^{-\beta \Phi(x)} d x\right)$.

Proof. According to Lemma 3.2 we have for every bounded Borel set $\Lambda \subset \mathbb{R}^{d}$

$$
\begin{aligned}
\mu\left(\Lambda^{*}\right) & \leqq \sum_{l=0}^{\infty} l !^{-1} z^{l+1} \int_{\Lambda} \int \ldots \int_{\left(\mathbb{R}^{d}\right)^{l}} e^{-\beta V\left(x, x_{1}, \ldots, x_{l}\right)} u\left(x, x_{1}, \ldots, x_{l}\right) d x_{1} \ldots d x_{l} \\
& =z \cdot \lambda(\Lambda) \sum_{l=0}^{\infty} l !^{-1} z^{l} \int \ldots \int_{\left(\mathbb{R}^{d}\right)^{l}} e^{-\beta V\left(x, x_{1}, \ldots, x_{l}\right)} u\left(x, x_{1}, \ldots, x_{l}\right) d x_{1} \ldots d x_{l}
\end{aligned}
$$

since the series (15) does not depend on $x$ because of the invariance under translations of $u$ and $V$ and

$$
\mu\left(\Lambda^{*}\right) \geqq z \cdot \lambda(\Lambda) \sum_{l=0}^{\infty}(l+1) !^{-1} z^{l} \int \ldots \int_{\left(\mathbb{R}^{d}\right)^{l}} e^{-\beta V\left(x, x_{1}, \ldots, x_{l}\right)} u\left(x, x_{1}, \ldots, x_{l}\right) d x_{1} \ldots d x_{l} .
$$

The upper and the lower bound are up to a factor $\lambda(\Lambda)$ power series in $z$ with the same radius of convergence. Thus the first part of Proposition 3.3 is proved.

For the second part we use the known results of the radius of convergence of the Mayer series ([6], Theorem 4.3.1).

Lemma 1.1 and the estimation

$e^{-\beta V\left(x, x_{1}, \ldots, x_{l}\right)} \leqq e^{\beta B(l+1)}$

yield the result for stable potentials.

From (1) there follows

$$
\begin{aligned}
& e^{-\beta V\left(x_{1}, \ldots, x_{l}\right)} u\left(x_{1}, \ldots, x_{l}\right) \\
& \quad=\sum_{G \in C_{l+1}} \prod_{(i, j) \in G}\left(1-h\left(x_{i}, x_{j}\right)\right) e^{-\beta \Phi\left(x_{j}-x_{l}\right)} \prod_{(a, b) \notin G} h\left(x_{a}, x_{b}\right) .
\end{aligned}
$$

For $\Phi \geqq 0$ we can define a potential $\varphi^{\prime}$ and the corresponding function $h^{\prime}$ by

$$
(1-h(x, y)) e^{-\beta \Phi(x-y)}=1-e^{-\beta \varphi^{\prime}(x-y)}=1-h^{\prime}(x, y) .
$$

Since $h \leqq h^{\prime}$ holds, there follows

$$
e^{-\beta V\left(x_{1}, \ldots, x_{l}\right)} u\left(x_{1}, \ldots, x_{l}\right) \leqq u^{\prime}\left(x_{1}, \ldots, x_{l}\right)
$$

with $u^{\prime}$ defined by replacing $h$ by $h^{\prime}$ in (1). The proof of Lemma 1.1 applies to this case, too, yielding

$$
u^{\prime}\left(x_{1}, \ldots, x_{l}\right) \leqq(-1)^{l-1} U^{\prime}\left(x_{1}, \ldots, x_{l}\right)
$$

with the Ursell function $U^{\prime}$ with respect to $\varphi^{\prime}$. From this the sharper result for non-negative potentials follows.

For the purpose of deriving cluster distributions corresponding to equilibrium particle distributions without infinite clusters with probability 1 we have to introduce the space of cluster configurations. The notions are the same as for particle configurations. So we only have to state the symbols we use for them. 
We call $\mathscr{Y}$ the set of all locally finite labelled configurations in $\mathscr{C}$ and $[\mathscr{Y}]$ the set of all locally finite non-labelled configurations in $\mathscr{C}$ with the corresponding topology. Furthermore we have for Borel sets $A \subset \mathscr{C}$ the sets $\mathscr{Y}_{A}$ and $\left[\mathscr{Y}_{A}\right]$ and the mapping $\Pi_{A}:[\mathscr{Y}] \rightarrow\left[\mathscr{Y}_{A}\right]$.

Let us consider now an equilibrium distribution $P$ on $[\mathscr{X}]$, such that there exist only finite clusters $P$-a.s. We want to describe the corresponding probability measure $Q$ on [Y] by its conditional distributions of clusters in $\Lambda_{*}$ under configurations outside $\Lambda_{*}$ by means of equations analogous to the DLR-equations.

We proceed from the DLR-equation (11) for a bounded Borel-set $\Lambda \subset \mathbb{R}^{d}$. Let us first fix the configuration $\left[\left(y_{i}\right)\right] \in\left[\mathscr{X}_{[\Lambda}\right]$. Then to the measure $P_{\Lambda \mid\left[\left(y_{i}\right)\right]}$ there corresponds the measure $\bar{Q}_{\Lambda_{*}\left[\left(y_{i}\right)\right]}$ on $\sum_{n=0}^{\infty}\left(\Lambda_{*}\right)_{\text {symm }}^{n}=\left[\mathscr{Y}_{\Lambda_{*}}\right]$ defined by (14). We shall separate each cluster configuration of $\left[\mathscr{Y}_{A^{*}}\right]$ into clusters interacting with the boundary particles and those which do not.

We denote the interaction region of an arbitrary particle configuration $\left[\left(x_{i}\right)\right] \in[\mathscr{X}]$ by

$$
i\left(\left[\left(x_{i}\right)\right]\right)=\bigcup_{i}\left\{x: h\left(x, x_{i}\right)=0\right\} \subset \mathbb{R}^{d}
$$

and analogously for cluster configurations $\left[\left(X_{k}\right)\right] \in[\mathscr{Y}]$

$$
I\left(\left[\left(X_{k}\right)\right]\right)=\bigcup_{k}\left\{X: H\left(X, X_{k}\right)=0\right\} \subset \mathscr{C} .
$$

If $\left[\left(X_{k}\right)\right] \in[\mathscr{Y}]$ and $\left[\left(x_{i}\right)\right] \in[\mathscr{X}]$ is the configuration of the particles belonging to all clusters $X_{k}$, then

$$
I\left(\left[\left(X_{k}\right)\right]\right)=i\left(\left[\left(x_{i}\right)\right]\right)^{*} .
$$

With this notion the above-mentioned separation of clusters corresponds to the identification of $\left[\mathscr{Y}_{\Lambda *}\right]$ with

$$
\left[\mathscr{Y}_{\Lambda_{*} \cap i\left(\left[\left(y_{i}\right)\right]\right)^{*}}\right] \times\left[\mathscr{Y}_{\Lambda_{*} \cap\left[i\left(\left[\left(y_{i}\right)\right]\right)^{*}\right.}\right]
$$

as measurable spaces equipped with their Borel sets.

The distribution of clusters in $\Lambda_{*}$ interacting with $\left[\left(y_{i}\right)\right]$ under $\bar{Q}_{\Lambda_{*}\left[\left[\left(y_{i}\right)\right]\right.}$ is now derived by regarding $\bar{Q}_{\Lambda_{*} \mid\left[\left(y_{i}\right)\right]}$ on (19) and integrating over the second factor. The result is the probability measure $\bar{Q}_{\Lambda_{*} \mid\left[\left(y_{i}\right)\right]}^{1}$ on $\left[\mathscr{Y}_{\Lambda_{*} \cap i\left(\left[\left(y_{i}\right)\right]\right)^{*}}\right]$ with the following density $\bar{q}_{\Lambda_{*} \mid\left[\left(y_{i}\right)\right]}^{1}$ with respect to $\not\left(\mu_{\Lambda_{*} \cap i\left(\left[\left(y_{2}\right)\right]\right)^{*}}\right)$ :

$$
\begin{aligned}
& \bar{q}_{\Lambda_{*} \mid\left[\left(y_{2}\right)\right]}^{1}\left(\left[\left(Z_{1}, \ldots, Z_{j}\right)\right]\right) \\
&=\overline{\mathcal{3}}\left(\Lambda_{*} \mid\left[\left(y_{i}\right)\right]\right)^{-1} j !^{-1} \sum_{k=0}^{\infty} k !^{-1} \int \ldots \int_{\left(\Lambda_{*} \cap C i\left(\left[\left(y_{i}\right)\right]\right)^{*)^{k}}\right.} H\left(Z_{1}, \ldots, Z_{j}, X_{1}, \ldots, X_{k}\right) \\
& \quad \cdot \prod_{m=1}^{j} \prod_{i} e^{-\beta W\left(Z_{m}, y_{i}\right)} d \mu\left(X_{1}\right) \ldots d \mu\left(X_{k}\right) \\
&=\overline{3}\left(\Lambda_{*} \mid\left[\left(y_{i}\right)\right]\right)^{-1} j !^{-1} H\left(Z_{1}, \ldots, Z_{j}\right) \prod_{m=1}^{j} \prod_{i} e^{-\beta W\left(Z_{m}, y_{i}\right)} \cdot \sum_{k=0}^{\infty} k !^{-1} \\
& \quad \cdot \int \ldots \int_{\left(\Lambda_{*} \cap C i\left(\left[\left(y_{i}\right)\right]\right)^{*}\right)^{k}} H\left(X_{1}, \ldots, X_{k}\right) \prod_{m=1}^{j} \prod_{n=1}^{k} H\left(Z_{m}, X_{n}\right) d \mu\left(X_{1}\right) \ldots d \mu\left(X_{k}\right) \\
&=\overline{3}\left(\Lambda_{*} \mid\left[\left(y_{i}\right)\right]\right)^{-1} j !^{-1} H\left(Z_{1}, \ldots, Z_{j}\right) \prod_{m=1}^{j} \prod_{i} e^{-\beta W\left(Z_{m}, y_{i}\right)} \\
& \cdot 3\left(\Lambda_{*} \cap C i\left(\left[\left(y_{i}\right)\right]\right)^{*} \cap C I\left(\left[\left(Z_{1}, \ldots, Z_{j}\right)\right]\right)\right) .
\end{aligned}
$$

Comparing (20) with (14) one gets $\bar{Q}_{\Lambda_{*}\left[\left[\left(y_{i}\right)\right]\right.}$ by $\bar{Q}_{\Lambda_{*}\left[\left[\left(y_{i}\right)\right]\right.}^{1}$ on the first factor of (19) and the following conditional distribution $\bar{Q}_{\Lambda_{*}\left[\left[\left(y_{i}\right)\right],\left[\left(Z_{1}, \ldots, Z_{j}\right)\right]\right.}^{2}$ on the second factor for fixed $\left[\left(Z_{1}, \ldots, Z_{j}\right)\right]: \bar{Q}_{A_{*} \mid\left[\left(y_{i}\right)\right],\left[\left(Z_{1}, \ldots, Z_{j}\right)\right]}^{2}$ has the density $\bar{q}_{\Lambda_{*} \mid\left[\left(y_{i}\right)\right],\left[\left(Z_{1}, \ldots, Z_{j}\right)\right]}^{2}$ with 
respect to $\not h\left(\mu_{A * \cap \subset i\left(\left[\left(y_{i}\right)\right]\right) *}\right)$ :

$$
\begin{aligned}
& \bar{q}_{\Lambda_{*} \mid\left[\left(y_{i}\right)\right],\left[\left(Z_{1}, \ldots, Z_{j}\right)\right]}\left(\left[\left(X_{1}, \ldots, X_{k}\right)\right]\right) \\
& \quad=3\left(\Lambda_{*} \cap \mathcal{C}\left(\left[\left(y_{i}\right)\right]\right)^{*} \cap \mathcal{C} I\left(\left[\left(Z_{1}, \ldots, Z_{j}\right)\right]\right)\right)^{-1} k !^{-1} H\left(X_{1}, \ldots, X_{k}\right) .
\end{aligned}
$$

Until now we kept $\left[\left(y_{i}\right)\right] \in\left[\mathscr{X}_{C_{\Lambda}}\right]$ fixed. Now we return to the DLR-equation (11). If we regard a particle configuration in $\mathbb{R}^{d}$ and subdivide it into clusters, then the clusters in $C \Lambda_{*}$ consist of the particles belonging to $C \Lambda$ and those in $\Lambda$, which cluster with particles in $\complement \Lambda$. Thus the distribution $\pi\left(P_{\complement A}\right)$ and the conditional distributions $\bar{Q}_{\Lambda_{*} \mid}^{1}$. lead to the distribution $\Pi_{\complement \Lambda_{*}}(Q)$ of clusters in $\complement \Lambda_{*}$.

If $\left[\left(y_{i}\right)\right] \in\left[\mathscr{X}_{C_{\Lambda}}\right]$ and $\left[\left(Z_{1}, \ldots, Z_{j}\right)\right] \in\left[\mathscr{Y}_{\left.\Lambda * \cap i\left(\left[\left(y_{i}\right)\right]\right]^{*}\right]}\right.$ are composed in this way to $\left[\left(Y_{m}\right)\right] \in\left[\mathscr{Y}_{C \Lambda_{*}}\right]$, then $(21)$ can be simplified to

$$
3\left(\Lambda_{*} \cap C I\left(\left[\left(Y_{m}\right)\right]\right)\right)^{-1} k !^{-1} H\left(X_{1}, \ldots, X_{k}\right)
$$

and we are led to the conditional distribution $Q_{\Lambda_{*}\left[\left(Y_{m}\right)\right]}$ on $\left[\mathscr{Y}_{\Lambda_{*}}\right]$ with the density $q_{\Lambda_{*} \mid\left[\left(Y_{m}\right)\right]}$ with respect to $\not\left(\mu_{\Lambda_{*}}\right)$

$$
\begin{aligned}
& q_{\Lambda_{*} \mid\left[\left(Y_{m}\right)\right]}\left(\left[\left(X_{1}, \ldots, X_{k}\right)\right]\right) \\
& \quad=3\left(\Lambda_{*} \cap C I\left(\left[\left(Y_{m}\right)\right]\right)\right)^{-1} k !^{-1} H\left(X_{1}, \ldots, X_{k}\right) \mathbb{1}_{C I\left(\left[\left(Y_{m}\right)\right]\right)^{k}}\left(X_{1}, \ldots, X_{k}\right) .
\end{aligned}
$$

1.denotes the indicator function of a set.

Inserting the different distributions derived above into the DLR-equation (11) we finally get the following cluster-DLR-equations:

For every bounded Borel set $\Lambda \subset \mathbb{R}^{d}$ and every $F \in L^{1}([\mathscr{Y}], Q)$

$$
\begin{aligned}
& \int_{[\mathscr{Y}]} F d Q=\int_{\left[\mathscr{Y}_{\left[\Lambda_{*}\right]}\right.} d \Pi_{\left[\Lambda_{*}\right.}(Q)\left(\left[\left(Y_{m}\right)\right]\right) \int_{\left[\mathscr{Y}_{\left.\Lambda_{*}\right]}\right.} d Q_{\Lambda_{*} \mid\left[\left(Y_{m}\right)\right]}\left(\left[\left(X_{1}, \ldots, X_{k}\right)\right]\right) \\
& F\left(\left[\left(Y_{m}\right)\right] \times\left[\left(X_{1}, \ldots, X_{k}\right)\right]\right) .
\end{aligned}
$$

Before we prove that conversely the cluster-DLR-equations (23) imply the DLR-equations (11), we have to show that they imply cluster-DLR-equations for more general sets.

So we define for a Borel set $A \subset \mathscr{C}$ with $\mu(A)<\infty$ and $\left[\left(Y_{m}\right)\right] \in\left[\mathscr{Y}_{C_{A}}\right]$ the con-

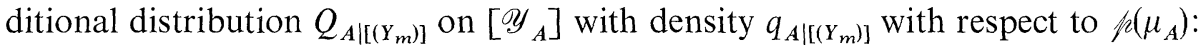

$$
\begin{aligned}
& q_{A \mid\left[\left(Y_{m}\right)\right]}\left(\left[\left(X_{1}, \ldots, X_{k}\right)\right]\right) \\
& \quad=3\left(A \cap C I\left(\left[\left(Y_{m}\right)\right]\right)\right)^{-1} k !^{-1} H\left(X_{1}, \ldots, X_{k}\right) \mathbb{1}_{C I\left(\left[\left(Y_{m}\right)\right]\right)^{k}}\left(X_{1}, \ldots, X_{k}\right) .
\end{aligned}
$$

The general cluster-DLR-equations are:

For every Borel set $A \subset \mathscr{C}$ with $\mu(A)<\infty$ and every $F \in L^{1}([\mathscr{Y}], Q)$

$$
\begin{aligned}
\int_{[\mathscr{Y}]} F d Q= & \int_{[\mathscr{Y} C A]} d \Pi_{C A}(Q)\left(\left[\left(Y_{m}\right)\right]\right) \int_{\left[\mathscr{Y}_{A}\right]} d Q_{A \mid\left[\left(Y_{m}\right)\right]}\left(\left[\left(X_{1}, \ldots, X_{k}\right)\right]\right) \\
& \cdot F\left(\left[\left(Y_{m}\right)\right] \times\left[\left(X_{1}, \ldots, X_{k}\right)\right]\right) .
\end{aligned}
$$

Let $Q$ be a probability measure on [Y] which satisfies (23). First we treat the case of a Borel set $A \subset \Lambda_{*}$ for a certain bounded Borel set $\Lambda \subset \mathbb{R}^{d}$. Separating the configurations in $\Lambda_{*}$ into clusters, which belong to $A$, and those, which do not, the same procedure as above shows that $\left(23^{\prime}\right)$ is satisfied for these $A$.

In the general case of a Borel set $A \subset \mathscr{C}$ with $\mu(A)<\infty$ we take a sequence of bounded Borel sets $\Lambda_{n} \uparrow \mathbb{R}^{d}$. Then (23') holds for $\Lambda_{n *} \cap A$ and a convergence theorem for conditional distributions ([4]) yields $\left(23^{\prime}\right)$ for $A$. 
Since $\left(23^{\prime}\right)$ implies (23) as a special case, thus (23) and (23') are equivalent. A probability measure $Q$ on [Y] satisfying (23) is called an equilibrium cluster distribution.

Let us consider now an equilibrium cluster distribution $Q$. We dissolve the clusters into particles and get a corresponding probability measure $P$ on $[\mathscr{X}]$. Let $\Lambda \subset \mathbb{R}^{d}$ be a bounded Borel set. Since $\mu\left(\Lambda_{\leq N}^{*}\right)<\infty$ for every $N,\left(23^{\prime}\right)$ holds for $\Lambda_{\leqq}^{*}$. We separate the particles of $\Lambda_{\leqq N}^{*}$ into particles, which are in $\Lambda$, and those which are not. Our familiar procedure yields DLR-equations for the particles in $A$ belonging to a cluster of $\Lambda_{\leq N}^{*}$. The DLR-equation (11) follows from the limit $N \rightarrow \infty$.

We thus proved the following theorem:

Theorem 3.4. Let $P$ be a probability measure on $[\mathscr{X}]$, such that there exist only finite clusters $P$-a.s., and let $Q$ be the corresponding probability measure on [Y]. Then $P$ satisfies the DLR-equations (11), iff $Q$ satisfies the cluster-DLR-equations (23).

The cluster-DLR-equations are formally the same as the DLR-equations, when we use again the measure $\mu$ and the hard core potential excluding overlapping clusters. Likewise they suggest the notion of a Poisson point process with exclusion, though $Q$ cannot be written directly as a conditional distribution, since the condition would have probability 0 .

\section{Existence of Equilibrium Cluster Distributions}

The formal agreement of the cluster-DLR-equations with the DLR-equations by use of a hard core potential suggests to transfer Dobrushin's methods ([1]) for an existence theorem to our case.

For this purpose we need some preparations.

Lemma 4.1. Let (13) be satisfied and $\Lambda \subset \mathbb{R}^{d}$ be a bounded Borel set. Then

$\int\left|q_{\Lambda^{*} \mid\left[\left(Y_{m}^{(1)}\right)\right]}-q_{\Lambda^{*} \mid\left[\left(Y_{m}^{(2)}\right)\right]}\right| d p\left(\mu_{\Lambda^{*}}\right)=O\left(\mu\left(\Lambda^{*} \cap I\left(\left[\left(Y_{m}^{(1)}\right)\right]\right) \Delta I\left(\left[\left(Y_{m}^{(2)}\right)\right]\right)\right)\right)$ for $\mu\left(\Lambda^{*} \cap I\left(\left[\left(Y_{m}^{(1)}\right)\right]\right) \Delta I\left(\left[\left(Y_{m}^{(2)}\right)\right]\right)\right) \rightarrow 0$.

Proof. Let $\left[\left(Y_{m}^{(1)}\right)\right],\left[\left(Y_{m}^{(2)}\right)\right] \in\left[\mathscr{Y}_{C \Lambda^{*}}\right]$ and set $I^{(j)}=I\left(\left[\left(Y_{m}^{(j)}\right)\right]\right)_{(j=1,2)}$.

In the case $I^{(1)} \subset I^{(2)}$ we have

$$
\begin{aligned}
& \int\left|q_{\Lambda^{*} \mid\left[\left(Y_{m}^{(1)}\right)\right]}-q_{\Lambda^{*} \mid\left[\left(Y_{m}^{(2)}\right)\right]}\right| d p\left(\mu_{\Lambda^{*}}\right) \\
&= \sum_{k=0}^{\infty} k !^{-1} \int \ldots \int_{\Lambda^{* k}} \mid 3\left(\Lambda^{*} \cap C I^{(1)}\right)^{-1} \mathbb{1}_{\left[\complement I^{(1)}\right]^{k}}\left(X_{1}, \ldots, X_{k}\right) \\
&-3\left(\Lambda^{*} \cap C I^{(2)}\right)^{-1} \mathbb{1}_{\left[C I^{(2)}\right]^{k}}\left(X_{1}, \ldots, X_{k}\right) \mid H\left(X_{1}, \ldots, X_{k}\right) d \mu\left(X_{1}\right) \ldots d \mu\left(X_{k}\right) \\
& \leqq \sum_{k=0}^{\infty} k !^{-1} \int \ldots \int_{\left(\Lambda^{*} \cap C I^{(2)}\right)^{k}}\left|3\left(\Lambda^{*} \cap C I^{(1)}\right)^{-1}-3\left(\Lambda^{*} \cap C I^{(2)}\right)^{-1}\right| \\
& \cdot H\left(X_{1}, \ldots, X_{k}\right) d \mu\left(X_{1}\right) \ldots d \mu\left(X_{k}\right) \\
&+\sum_{k=0}^{\infty} k !^{-1} \int_{\Lambda^{*} \cap\left(I^{(2)} \backslash I^{(1)}\right)} \int \ldots \int_{\left(\Lambda^{*} \cap C I^{(1)}\right)^{k}} 3\left(\Lambda^{*} \cap C I^{(1)}\right)^{-1} \\
& \cdot H\left(X, X_{1}, \ldots, X_{k}\right) d \mu(X) d \mu\left(X_{1}\right) \ldots d \mu\left(X_{k}\right) \\
& \leqq\left(1-e^{-\mu\left(\Lambda^{*} \cap\left(I^{(2)} \backslash I^{(1)}\right)\right.}\right)+\mu\left(\Lambda^{*} \cap\left(I^{(2)} \backslash I^{(1)}\right)\right) .
\end{aligned}
$$

We used for (24) Lemma 3.2 and for (25) Lemma 2.1 iii) and then i).

In the general case we introduce $I=I^{(1)} \cup I^{(2)}$. Applying the special case to $I^{(1)} \mathrm{C} I$ and $I^{(2)} \mathrm{C} I$ the result follows. 
Corollary 4.2. Under the assumptions of Lemma 4.1 the set of all densities

$q_{\Lambda^{*} \mid\left[\left(Y_{m}\right)\right]}$ with $\left[\left(Y_{m}\right)\right] \in\left[\mathscr{Y}_{\left[\Lambda^{*}\right.}\right]$ is precompact in $L^{1}\left(\left[\mathscr{Y}_{\Lambda^{*}}\right], f\left(\mu_{\Lambda^{*}}\right)\right)$.

Proof. For $\varepsilon>0$ and $N>0$ we consider a finite $\varepsilon$-net in $\{x: x \notin \Lambda, \operatorname{dist}(x, \Lambda) \leqq N\} C$ $\mathbb{R}^{d}$. We subdivide every subset of it into clusters. Thus we get a finite set of configurations of $\left[\mathscr{Y}_{C \Lambda^{*}}\right]$. Lemma 4.1 shows that for $\delta>0$ there exist $\varepsilon>0$ and $N>0$ such that the corresponding densities $q_{\Lambda^{*} \mid}$. form a finite $\delta$-net in $L^{1}\left(\left[\mathscr{Y}_{\Lambda^{*}}\right], h\left(\mu_{\Lambda^{*}}\right)\right)$.

Corollary 4.3. Under the assumptions of Lemma 4.1 and for $\Lambda \subset \Lambda_{n} \uparrow \mathbb{R}^{d}$

$$
\sup \left\{\int\left|q_{\Lambda^{*} \mid\left[\left(Y_{m}^{(1)}\right)\right]}-q_{\Lambda^{*} \mid\left[\left(Y_{m}^{(2)}\right)\right]}\right| d \not p\left(\mu_{\Lambda^{*}}\right): \Pi_{\Lambda_{n}^{*} \backslash \Lambda^{*}}\left(\left[\left(Y_{m}^{(1)}\right)\right]\right)=\Pi_{\Lambda_{n}^{*} \backslash \Lambda^{*}}\left(\left[\left(Y_{m}^{(2)}\right)\right]\right)\right\} \rightarrow 0
$$
for $n \rightarrow \infty$.

Proof. Clear.

Now the proof of Theorem 1 of [1a] can be transferred directly. Thus we briefly outline the proof.

There follows from Corollary 4.2 that for a bounded Borel set $\Lambda \subset \mathbb{R}^{d}$ the closed convex hull $\mathfrak{A}_{\Lambda^{*}}$ of all densities $q_{\Lambda^{*} \mid}$. in $L^{1}\left(\left[\mathscr{Y}_{\Lambda^{*}}\right], \not\left(\mu_{\Lambda^{*}}\right)\right)$ is compact. We equip the set of all probability measures $Q$ on $[\mathscr{Y}]$, such that $\Pi_{\Lambda^{*}}(Q)$ has a density with respect to $h\left(\mu_{\Lambda^{*}}\right)$ for every bounded Borel set $\Lambda \subset \mathbb{R}^{d}$, with the projective topology of the $L^{1}\left(\left[\mathscr{Y}_{\Lambda^{*}}\right], h\left(\mu_{\Lambda^{*}}\right)\right)$-topologies of these densities. Taking a sequence $\Lambda_{n} \uparrow \mathbb{R}^{d}$ the intersection $\bigcap_{n} \mathfrak{A}_{\Lambda_{n}^{*}}$ is non-empty and coincides with the closed convex hull of probability measures which are limits of $Q_{n_{k}} \in \mathfrak{U}_{\Lambda_{n_{k}}^{*}}$ with an arbitrary subsequence $\mathfrak{H}_{\Lambda_{n_{k}^{*}}^{*}}$.

Because of Corollary 4.3 and by means of the separation method this is the set of all equilibrium cluster distributions. The statement of Theorem 1 of [1a] concerning translational invariant equilibrium cluster distributions is likewise transferred, if we introduce on the cluster space $\mathscr{C}$ translations with elements of $\mathbb{R}^{d}$ by translating all particles of the clusters.

Thus we have:

Theorem 4.4. Let (13) be satisfied. Then the set of all equilibrium cluster distributions is a non-empty closed convex set coinciding with the closed convex hull of the set of distributions which are limits of conditional distributions $q_{\Lambda * \mid}$. with different boundary conditions with bounded Borel sets $\Lambda \uparrow \mathbb{R}^{d}$. The set of all translational invariant equilibrium cluster distributions is a non-empty closed set, too.

Because of Theorem 3.4 we have the following corollary.

Corollary 4.5. Let (13) be satisfied. Then the set of all equilibrium particle distributions $P$, such that there exist only finite clusters $P$-a.s., is a non-empty convex set. The set of all such translational invariant equilibrium distributions is a non-empty convex set, too.

\section{Final Remarks}

To derive uniqueness theorems for equilibrium cluster distributions there are two possibilities. The first one is to transfer again methods for equilibrium particle distributions (Theorem 6 of [1a]) the second one is to apply Theorem 3.4 and use directly uniqueness theorems for equilibrium particle distributions, which are 
known for more general cases than existence theorems ([6], Section 4.2). The second way leads to the uniqueness for small activity. Though this condition ([6], 4.2.33) and the condition of Proposition 3.3 for the validity of (13) are not comparable, we conjecture the following: The condition (13) is necessary and sufficient for an equilibrium distribution $P$ to have only finite clusters $P$-a.s. the passage to infinite clusters thus occuring at the radius of convergence of (15). In the case of finite clusters the equilibrium particle distribution and hence the equilibrium cluster distribution is unique. The first conjecture is suggested among others by the independent use of (13) in Theorems 3.1 and 4.4. Remark at this opportunity that the proof of Theorem 4.4 does not work by replacing $\Lambda^{*}$ by $\Lambda_{*}$ because of the influence of the outer configurations.

Note Added in Proof. After printing of the article we proved part of the conjectures in Section 5, namely that condition (13) implies the uniqueness of the equilibrium cluster distribution (to be published).

\section{References}

1. Dobrushin, R. L.: Funkts. Anal. Ego Pril. a) 2, 31-43 (1968); b) 2, $44-57$ (1968); c) 3, 27-35 (1969)

2. Groeneveld, J.: Estimation methods for Mayer's graphical expansions. In: Harary, F. (Ed.): Graph theory and theoretical physics, pp. 229-259. London: Academic Press 1967

3. Hill, T.L.: Statistical mechanics. New York: McGraw-Hill 1956

4. Hunt, G. A.: Martingales et processus de Markov. Paris: Dunod 1966

5. Lanford, O. E.: Commun. math. Phys. 11, 257-292 (1969)

6. Ruelle, D.: Statistical mechanics. New York: Benjamin 1969

7. Ruelle, D.: Commun. math. Phys. 18, 127-159 (1970)

8. Sinai, Y. G.: Teor. i Mat. Fiz. 11, 248-258 (1972)

Communicated by G. Gallavotti

(Received June 9, 1975) 